\title{
Ischemic Stroke in Young Adults of Northern China: Characteristics and Risk Factors for Recurrence
}

\author{
Fang $\mathrm{Li}^{\mathrm{a}}$ Li Yang ${ }^{\mathrm{a}}$ Rui Yang ${ }^{\mathrm{a}}$ Wei Xu ${ }^{\mathrm{a}}$ Fu-ping Chen ${ }^{\mathrm{a}}$ Nan Li ${ }^{\mathrm{b}}$ \\ Jin-biao Zhang ${ }^{c}$ \\ ${ }^{a}$ Department of Neurology, The First Affiliated Hospital of Jinzhou Medical University, and bepartment of \\ Neurology, Jinzhou Central Hospital, Jinzhou, and 'Department of Neurology, Weihai Municipal Hospital, \\ The Affiliated Hospital of Binzhou Medical College, Weihai, PR China
}

\section{Key Words}

Stroke $\cdot$ Young adult $\cdot$ Risk factor · Etiology ·

Disease severity $\cdot$ Recurrence

\begin{abstract}
Background: Young adults accounted for $10-14 \%$ of ischemic stroke patients. The risk factors may differ in this population from elder patients. In addition, the factors associated with stroke recurrence in this population have not been well investigated. Objective: The study aimed to investigate the characteristics and risk factors associated with recurrence of ischemic stroke in young adults. Methods: Clinical data of 1,395 patients of age 18-45 years who were treated between 2008 and 2014 in 3 centers located in northern China was reviewed. The first onset of stroke was taken as the initial events and recurrent stroke as the end point events. The end point events, age, gender, duration after first onset of stroke, history of disease, National Institutes of Health Stroke Scale (NIHSS) score at admission, Trial of Org 10172 in Acute Stroke Treatment classifications of the cause of stroke and adherence to medication were recorded. These factors were analyzed and compared between recurrence and non-recurrence group. Information about recurrent stroke was collected through clinical (readmission to hospital with ischemic stroke) or telephone follow-up survey. Logistic regression
\end{abstract}

was used to analyze the risk factors of recurrence. Results: The most common causes of stroke were large vessel atherosclerosis and small vessel occlusion, followed by cardioembolism. NIHSS score at admission (OR 1.088; $95 \%$ Cl 1.0281.152; $p=0.004$ ) were associated with recurrence. Conclusions: Vascular disease, especially premature atherosclerosis, is the major risk factor for ischemic stroke in the young adult population of northern China. Timely screening of the cause of stroke with severe NIHSS score needs further attention.

(c) 2017 The Author(s)

Published by S. Karger AG, Basel

\section{Introduction}

Ischemic stroke in young adults is defined as acute ischemic cerebrovascular disease that occurs in patients aged 18-45 years [1]. Approximately $10-14 \%$ of ischemic strokes occur in young adults [2]. Although the shortterm prognosis of this population is better than in older patients, ischemic stroke can have lasting detrimental effects in the lives of young adults [3]; with cumulative mortality rate ranging $1-7 \%$ in 5 years [4]. Furthermore, young adults may suffer long-term dysfunction after having suffered a stroke. Understanding of factors associated with the occurrence and recurrence of stoke in these patients would help clinical prevention and intervention.

\section{KARGER}

E-Mail karger@karger.com www.karger.com/ene

\section{The Author(s) \\ Published by S. Karger AG, Basel \\ Karger \\ Open access}

This article is licensed under the Creative Commons AttributionNonCommercial-NoDerivatives 4.0 International License (CC BYNC-ND) (http://www.karger.com/Services/OpenAccessLicense). Usage and distribution for commercial purposes as well as any distribution of modified material requires written permission.
Department of Neurology, Weihai Municipal Hospital

The Affiliated Hospital of Binzhou Medical College

70th Heping Street, Weihai 264200, Shandong (PR China)

E-Mail drzhangjinbiao@163.com 
Previous studies have been undertaken to examine the risk factors associated with initial and recurrent ischemic stroke in young adults [5]. Burden of vascular risk factors, family history of cardiovascular disease, and increased intima-media thickness of the internal carotid artery have been reported to be risk factors for ischemic stroke in young and middle aged adults (15-60 years) [6]. It has also been reported that certain genetic mutation (prothrombin G20210A) was associated with ischemic stroke in young adults [7]. A review of data from population-based studies in the United States identified increasing prevalence of stroke risk factors among persons aged 15-44 years of age, including hypertension, diabetes mellitus, obesity, lipid disorders, congenital heart disease, and smoking [8].

The available studies with respect to populations and ethnicity are still limited. The potential factors related with the severity of stroke have not been considered. Specifically, there is very limited data available on the causes and risk factors of ischemic stroke in Chinese young adults. Therefore, the current study aims to investigate the characteristics and recurrence risk factors for ischemic stroke in young adults, as well as their relations with disease severity.

\section{Methods}

\section{Patients}

We retrospectively reviewed data of patients of age $18-45$ years who were treated for ischemic stroke between 2008 and 2014 at 3 stroke centers in northern China. Ischemic stroke was defined as a sudden neurological deficit, and the infarct site was assessed by MRI. Symptoms onset include sudden weakness, numbness, or loss of control of face, arm, or leg on one side of the body, sudden dimness, or loss of vision in one or both eyes, loss of speech, or trouble talking and understanding speech, dizziness, unsteadiness, or sudden fall and difficulty in wallowing. Patients with the following were excluded from the study: intracranial venous thrombosis, transient ischemic attack, cerebral hemorrhage, and brain tumors. The study was approved by the Human Research Committee of the First Affiliated Hospital of Jinzhou Medical University, China.

\section{Data Collection}

All patients completed the follow-up for at least 1 year. The median recurrence time of the patients was 231 days (ranged from 21 to 734 days). Medical records of study subjects were reviewed by 2 senior physicians from the Department of Neurology. Demographic data, medical history, and family history (hypertension, diabetes mellitus, and stroke) were extracted. In addition, disease severity was categorized based on National Institutes of Health Stroke Scale (NIHSS) score as mild (0-6), moderate (7-14), or severe $(\geq 15)$. Etiology was categorized according to the Trial of Org 10172 in Acute Stroke Treatment (TOAST) classification as large artery atherosclerosis, cardioembolism, small vessel occlusion, other determined etiology, and undetermined etiology. NIHSS score and TOAST classification were extracted from clini- cal records when available. In cases (53 of 1,395 cases, 3.8\%) not evaluated according to the original records, they were re-assessed by the physician who reviewed records based on the data available.

Total cholesterol, low-density lipoprotein (LDL), high-density lipoprotein, fasting plasma glucose, and glycosylated hemoglobin levels were measured by standard laboratory methods. Homocysteine levels were assayed by stable isotope dilution gas chromatography-mass spectrometry. Hypersensitive C-reactive protein (hs-CRP) levels were determined using a latex-enhanced immunonephelometric method on a Hitachi 7600 autoanalyzer. Antinuclear antibody were assessed by indirect fluorescence, the type of antinuclear antibody was identified by immunoblotting. The results of diagnostic blood tests (e.g., total cholesterol and LDL) were classified as normal or abnormal according to the reference ranges of the laboratory of the First Affiliated Hospital of Jinzhou Medical University, China. The cardiac factors in clinical records included atrial fibrillation or atrial flutter assessed with electrocardiogram or dynamic electrocardiogram, and mural thrombus or congenital structural abnormalities observed on echocardiography. Infarct sites were determined using cranial MRI (T1-, T2-, and diffusionweighted sequences). Radiographic examination findings included stenosis or occlusion of the culprit vessel (specific vessel affected), poor fibromuscular dysplasia observed using vascular ultrasonography $(1,137 / 1,328,85.6 \%)$, transcranial Doppler ultrasonography $(1,137 / 1,328,85.6 \%)$, magnetic resonance angiography (MRA, $306 / 1,328,23.0 \%$ ), CT angiography (CTA, 138/1,328, 10.4\%), or digital subtraction angiography (DSA, 613/1,328, 46.2\%).

Recurrent stroke was defined as new persisting ( $\geq 24 \mathrm{~h}$ ) neurological deficit occurring $>24 \mathrm{~h}$ after the index event and not attributable to other causes of neurological deterioration. In this article, the data about recurrence of ischemic stroke were collected through clinical (readmission to hospital with ischemic stroke) or telephonic follow-up survey. The first onset of stroke was taken as the initial event, and recurrent stroke as the end point event. The end point events, age, gender, duration after first onset of stroke, history of disease, NIHSS score at admission, TOAST classifications of the cause of stroke, and adherence to medication were recorded.

\section{Statistical Analysis}

Statistical analysis was performed using SPSS software version 17.0. Descriptive statistics were analyzed for total subjects. Categorical data was expressed as frequency (percentage). Comparisons among groups were performed using the $\chi^{2}$ test. The risk factors associated with disease recurrence were analyzed using multivariate logistic regression analysis, including all factors that showed statistical significant differences between groups. Correlations between ranked variables were analyzed using Spearman rank correlation analysis. A value of $p \leq 0.05$ was considered statistically significant.

\section{Results}

\section{Baseline Demographic and Clinical Characteristics}

A total of 1,395 patients were included in this study, with a mean age of $38.50 \pm 6.318$ years (range $18-45$ years). The population included $1,104(79.1 \%)$ men aged $38.77 \pm$ 5.980 years and $291(20.9 \%)$ women aged $37.46 \pm 7.382$ 
Table 1. Baseline characteristics

\begin{tabular}{|c|c|}
\hline & Constituent ratio, $\%$ \\
\hline & total number $(n=1,395)$ \\
\hline \multicolumn{2}{|l|}{ Demographic data } \\
\hline Age, years, mean (SD) & $38.50(6.318)$ \\
\hline Gender, male & $1,104(79.1)$ \\
\hline \multicolumn{2}{|l|}{ History of disease } \\
\hline Hypertension & $620(44.4)$ \\
\hline Diabetes mellitus & $192(13.8)$ \\
\hline Abnormal lipid metabolism & $234(16.8)$ \\
\hline Valvular disease & $19(1.4)$ \\
\hline Cardiomyopathy & $7(0.5)$ \\
\hline Atrial fibrillation & $14(1.0)$ \\
\hline Other arrhythmia & $12(0.9)$ \\
\hline \multicolumn{2}{|l|}{ Atrial septal defect/patent } \\
\hline foramen ovale & $15(1.1)$ \\
\hline Myxoma & $2(0.1)$ \\
\hline Heart failure & $4(0.3)$ \\
\hline Angina & $23(1.6)$ \\
\hline Myocardial infarction & $14(1.0)$ \\
\hline Smoking & $657(47.1)$ \\
\hline Drinking & $581(41.6)$ \\
\hline \multicolumn{2}{|l|}{ History of transient ischemic } \\
\hline attack & $38(2.7)$ \\
\hline Cancer & $3(0.2)$ \\
\hline History of migraine & $18(1.3)$ \\
\hline Family history & $547(39.2)$ \\
\hline \multicolumn{2}{|l|}{ NIHSS severity at admission } \\
\hline Mild (0-6) & $1,066(76.4)$ \\
\hline Moderate (7-14) & $293(21.0)$ \\
\hline Severe $(\geq 15)$ & $22(1.6)$ \\
\hline Missing & $14(1.0)$ \\
\hline
\end{tabular}

Missing: data of NIHSS score in 14 patients was missing. NIHSS, National Institutes of Health Stroke Scale.

years. The median interval between onset of stroke to admission was $36 \mathrm{~h}$ (ranged from 4 to $156 \mathrm{~h}$ ) for all patients. Hypertension was detected in $44.4 \%$ of patients, positive family history in $39.2 \%$, abnormal lipid metabolism in $16.8 \%$, diabetes mellitus in $13.8 \%$, smoking in $47.1 \%$, and alcohol ingestion in $41.6 \%$. The medical history included transient ischemic attack in $2.7 \%$ of patients, coronary heart disease (angina or myocardial infarction) in $2.6 \%$, valvular heart disease in $1.4 \%$, headaches in $1.3 \%$, patent foramen ovale in $1.1 \%$, and atrial fibrillation in $1.0 \%$.

\section{NIHSS Score at Admission}

The severity of stroke according to the NIHSS score was recorded as mild in 1,066 (76.4\%) patients, moderate in $293(21.0 \%)$, severe in $22(1.6 \%)$, and undetermined because of missing data in $14(1.0 \%)$ patients (Table 1$)$.
Table 2. Results of diagnostic tests

\begin{tabular}{lc}
\hline Item & $\begin{array}{l}\text { Positive } \\
\text { rate }\end{array}$ \\
& \\
\hline Blood & \\
Total cholesterol $>200 \mathrm{mg} / \mathrm{dL}$ & $115 / 1,336$ \\
Low-density lipoprotein $>130 \mathrm{mg} / \mathrm{dL}$ & $99 / 1,336$ \\
High-density lipoprotein $<35 \mathrm{mg} / \mathrm{dL}$ & $408 / 1,336$ \\
Fasting plasma glucose $>7 \mathrm{mmol} / \mathrm{L}$ & $191 / 1,332$ \\
Glycosylated hemoglobin $>7$ mmol/L & $83 / 332$ \\
Homocysteine $>12$ mmol/L & $451 / 688$ \\
Antinuclear antibody & $23 / 498$ \\
ANCA & $18 / 602$ \\
LA & $10 / 348$ \\
aCL IgG & $6 / 348$ \\
aCL IgM & $7 / 348$ \\
hs-CRP $>1.2$ mg/L & $4 / 836$ \\
Echocardiography & \\
Mural thrombosis & $9 / 703$ \\
Patent foramen ovale/atrial septal aneurysm & $60 / 703$ \\
Valvular heart diseases & $9 / 703$ \\
Cardiomyopathy & $4 / 703$ \\
Atrial fibrillation & $9 / 940$ \\
Cranial MRI & $1,088 / 1,088$ \\
Cranial CT & $506 / 621$ \\
Vascular tests (CTA, MRA, DSA, vascular ultrasound) & $891 / 1,328$ \\
Anterior circulation & $587 / 1,328$ \\
Posterior circulation & $143 / 1,328$ \\
Both anterior and posterior circulations & $161 / 1,328$ \\
$\quad$ Moyamoya disease & $49 / 1,328$ \\
$\quad$ Artery dissection & $11 / 1,328$ \\
$\quad$ Poor muscle fiber development & $3 / 1,328$ \\
\hline & \\
& \\
&
\end{tabular}

DSA $(+)=550 ;$ MRA $(+)=229 ;$ CTA $(+)=91 ;$ vascular ultrasonography, culprit vessel $>50 \%$ stenosis $(+)=753$.

CTA, CT angiography; MRA, magnetic resonance angiography; DSA, digital subtraction angiography.

\section{Diagnostic Tests}

The diagnostic test results are shown in Table 2. Diagnostic tests (including blood tests, electrocardiogram or dynamic electrocardiogram, echocardiography, and MRI) were performed in every patient. Vascular ultrasonography and transcranial Doppler ultrasonography were performed in $85.6 \%$ patients, and other patients were observed using MRA, CTA, or DSA.

A high proportion of patients (44.6\%) had abnormal lipid metabolism. The majority of patients underwent the following tests: antinuclear antibody, anti-neutrophil cytoplasmic antibody (ANCA), lupus anticoagulants (LA), anti-cardiolipin antibody (aCL), b2-glycoprotein I antibody (b2GPI), and hs-CRP concentrations. Antinuclear antibodies and ANCA were detected in 41 patients, IgM aCL was positive in 7 patients, IgG aCL in 6 patients. 
Among of these 13 patients (IgM aCL or IgG aCL was positive), LA was positive in 10 cases. There were no b2GPI-positive patients. Abnormal hs-CRP was recorded in 4 patients.

Complete echocardiography data were available for 703 patients, including 60 with a patent foramen ovale (7 combined with an atrial septal aneurysm), 9 with valvular heart disease, 9 with mural thrombus, and 4 with cardiomyopathy. A low proportion of patients had atrial fibrillation on electrocardiography or dynamic electrocardiography (9/940, 1\%).

A high proportion of patients had abnormal angiography findings $(891 / 1,328,67 \%)$. The abnormal findings were detected on DSA in 550 patients, on MRA in 229 , on CTA in 91, and on vascular ultrasonography in 753. Vascular anomalies were detected in the internal carotid artery system in $587(44.2 \%)$ patients, in the vertebrobasilar system in $143(10.8 \%)$, and in both the internal carotid artery and vertebrobasilar systems in 161 (12.1\%). Forty-nine patients had Moyamoya disease ( 29 men and 20 women), and 11 had dissection of the culprit artery ( 9 men and 2 women) including 2 with Marfan syndrome, who also had vertebral artery dissection. Poor muscle fiber development was detected in 3 men.

\section{Causes of Stroke}

Etiology of stroke was assessed according to TOAST classifications. Large artery atherosclerosis and small vessel occlusion accounted for nearly $50 \%$ of all cases. Large artery atherosclerosis was detected in 596 (42.7\%) patients (including 244 extracranial and 352 intra acranial), small vessel occlusion in 225 (16.1\%), cardioembolism in $90(6.5 \%)$ (53 with patent foramen ovale, 7 with patent foramen ovale as well as atrial septal aneurysm, 6 with valvular disease, 7 with atrial fibrillation, 5 with valvular disease as well as atrial fibrillation, 4 with cardiomyopathy, 1 with angina, 1 with myocardial infarction, 2 with other arrhythmia, 2 with left atrial appendage thrombosis, 1 with acute infective endocarditis, and 1 with Keshan disease), other determined etiology in 84 (6.0\%) (49 with Moyamoya disease, 11 with dissection of the culprit artery, 3 with poor muscle fiber development, 3 with pregnancy-related stroke, 4 with multiple arteritis, and 1 with cerebral autosomal dominant arteriopathy with subcortical infarcts and leukoencephalopathy), 2 or more causes in 153 (11.0\%), negative evaluation in 204 (14.6\%) (29 with undetermined cause and 175 with incomplete data), and incomplete evaluation in 41 (2.9\%; Table 3).
Table 3. TOAST classifications of the cause of stroke

\begin{tabular}{lrr}
\hline TOAST classification & $n$ & $\begin{array}{l}\text { Constituent } \\
\text { ratio, \% }\end{array}$ \\
\hline Large artery atherosclerosis & 596 & 42.7 \\
Cardioembolic stroke & 90 & 6.5 \\
$\quad$ Patent foramen ovale & 53 & \\
Patent foramen ovale + atrial septal aneurysm & 7 & \\
Valvular disease & 6 & \\
Atrial fibrillation & 7 & \\
Valvular disease + atrial fibrillation & 5 & \\
Cardiomyopathy & 4 & \\
Angina & 1 & \\
Myocardial infarction & 1 & \\
Other arrhythmia & 2 & \\
Left atrial appendage thrombosis & 2 & \\
Acute infective endocarditis & 1 & \\
Keshan disease & 1 & \\
Small vessel disease & 225 & 16.1 \\
Other clear causes & 84 & 6.0 \\
Moyamoya disease & 49 & \\
Antiphospholipid syndrome & 13 & \\
Artery dissection & 11 & \\
Poor muscle fiber development & 3 & \\
Pregnancy-related stroke & 3 & \\
Polyarteritis & 4 & \\
CADASIL & 1 & \\
Multiple potential causes & 153 & 11.0 \\
Unclear cause + incomplete work up & 204 & 14.6 \\
Missing & 41 & 2.9 \\
\hline & & \\
\hline
\end{tabular}

TOAST, Trial of Org 10172 in Acute Stroke Treatment; CADASIL, cerebral autosomal dominant arteriopathy with subcortical infarcts and leukoencephalopathy.

\section{Characters and Risk Factors for Recurrence of Ischemic Stroke}

Among studied patients, a total of 94 (6.7\%) patients inclusive of 72 men (6.5\% in total male subjects) and 22 women ( $7.6 \%$ in female subjects), experienced recurrence of ischemic stroke. The average age was $37.98 \pm 5.30$ years, the average duration after first onset of stroke was 338.7 days; 81 patients adhered to medication, such as managing arterial hypertension, disorders of lipid metabolism, and diabetes mellitus, and administering antiplatelets, but adherence to anticoagulants was poor (Table 4).

Associations between the baseline characteristics and recurrent ischemic stroke were first analyzed using univariate logistic regression analyses. Factors showing significant association with recurrent ischemic stroke on univariate analysis $(p<0.1)$ were then included in the multivariate logistic regression analysis. Univariate regression analyses found that recurrence was associated with abnormal lipid metabolism (OR 0.321; 95\% CI 
Table 4. Recurrence event characters

\begin{tabular}{|c|c|c|c|}
\hline & $\begin{array}{l}\text { Recurrence group } \\
(n=94), \%\end{array}$ & $\begin{array}{l}\text { Non-recurrence group } \\
(n=1,301)\end{array}$ & $p$ value \\
\hline \multicolumn{4}{|l|}{ Demographic data } \\
\hline Age, years, mean $(\mathrm{SD})$ & $37.98 \pm 5.30$ & $38.53 \pm 4.98$ & 0.477 \\
\hline Gender, male & $72(6.5)$ & $1,032(93.5)$ & 0.411 \\
\hline Duration after first onset of stroke, days & 338.7 & & \\
\hline \multicolumn{4}{|l|}{ History of disease } \\
\hline Hypertension & & & 0.962 \\
\hline Yes & $42(6.8)$ & $578(93.2)$ & \\
\hline No & $52(6.7)$ & $723(93.3)$ & \\
\hline Diabetes & & & 0.342 \\
\hline Yes & $16(8.3)$ & $176(91.7)$ & \\
\hline No & $78(6.5)$ & $1,125(93.5)$ & \\
\hline \multicolumn{3}{|l|}{ Hyperlipidemia } & 0.404 \\
\hline Yes & $6(2.6)$ & $228(97.4)$ & \\
\hline No & $88(7.6)$ & $1,073(92.4)$ & \\
\hline \multicolumn{3}{|l|}{ Valvular heart disease } & $1.000^{*}$ \\
\hline Yes & $1(5.3)$ & $18(94.7)$ & \\
\hline No & $93(6.8)$ & $1,283(93.2)$ & \\
\hline \multicolumn{3}{|l|}{ Cardiomyopathy } & $0.482^{\#}$ \\
\hline Yes & $1(14.3)$ & $6(85.7)$ & \\
\hline No & $93(6.7)$ & $1,295(93.3)$ & \\
\hline \multicolumn{3}{|l|}{ Atrial fibrillation } & $0.280^{\#}$ \\
\hline Yes & $3(21.4)$ & $11(78.6)$ & \\
\hline No & $91(6.6)$ & $1,290(93.4)$ & \\
\hline \multicolumn{3}{|l|}{ Other arrythmia } & $0.239^{\#}$ \\
\hline Yes & $2(16.7)$ & $10(83.3)$ & \\
\hline No & $92(6.7)$ & $1,291(93.3)$ & \\
\hline \multicolumn{3}{|l|}{ Atrial septal defect/PFO } & $0.612^{*}$ \\
\hline Yes & $2(13.3)$ & $13(86.7)$ & \\
\hline \multirow{2}{*}{\multicolumn{3}{|c|}{$\begin{array}{c}\text { No } \\
\text { Myxoma }\end{array}$}} & \\
\hline & & & $0.597^{\#}$ \\
\hline Yes & $0(0.0)$ & $2(100.0)$ & \\
\hline No & $94(6.7)$ & $1,299(93.3)$ & \\
\hline \multicolumn{3}{|l|}{ Hear failure } & $0.455^{\#}$ \\
\hline Yes & $0(0.0)$ & $4(100.0)$ & \\
\hline No & $94(6.8)$ & $1,297(93.2)$ & \\
\hline \multicolumn{3}{|l|}{ Angina } & $0.425^{*}$ \\
\hline Yes & $3(13.0)$ & $20(87.0)$ & \\
\hline No & $91(6.6)$ & $1,281(93.4)$ & \\
\hline \multicolumn{3}{|l|}{ Myocardial infarction } & $0.952^{\#}$ \\
\hline Yes & $1(7.1)$ & $13(92.9)$ & \\
\hline No & $93(6.7)$ & $1,288(93.3)$ & \\
\hline \multicolumn{3}{|l|}{ Smoking } & 0.490 \\
\hline Yes & $35(5.3)$ & $622(94.7)$ & \\
\hline No & $59(8.0)$ & $679(92.0)$ & \\
\hline \multicolumn{3}{|l|}{ Drinking } & 0.265 \\
\hline Yes & $34(5.9)$ & $547(94.1)$ & \\
\hline No & $60(7.4)$ & $754(92.6)$ & \\
\hline \multicolumn{3}{|l|}{ Prior TIA } & $0.778^{\#}$ \\
\hline Yes & $3(7.9)$ & $35(92.1)$ & \\
\hline & $91(6.7)$ & $1,266(93.3)$ & \\
\hline \multicolumn{3}{|l|}{ Cancer } & $0.172^{\#}$ \\
\hline Yes & $1(33.3)$ & $2(66.7)$ & \\
\hline No & $93(6.7)$ & $1,299(93.3)$ & \\
\hline \multicolumn{3}{|l|}{ History of headache } & $0.500^{*}$ \\
\hline Yes & $0(0.0)$ & $18(100.0)$ & \\
\hline No & $94(6.8)$ & $1,283(93.2)$ & \\
\hline
\end{tabular}


Table 4. (continued)

\begin{tabular}{|c|c|c|c|}
\hline & $\begin{array}{l}\text { Recurrence group } \\
(n=94), \%\end{array}$ & $\begin{array}{l}\text { Non-recurrence group } \\
(n=1,301)\end{array}$ & $p$ value \\
\hline Family history & & & 0.453 \\
\hline Yes & $38(6.9)$ & $509(93.1)$ & \\
\hline No & $56(6.6)$ & $792(93.4)$ & \\
\hline NIHSS severity at admission & & & 0.004 \\
\hline Mild (0-6) & $63(5.9)$ & $1,003(94.1)$ & \\
\hline Moderate (7-14) & $29(9.9)$ & $264(90.1)$ & \\
\hline Severe $(\geq 15)$ & $2(9.1)$ & $20(90.9)$ & \\
\hline Missing & $0(0.0)$ & $14(100)$ & \\
\hline TOAST classifications of the cause of stroke & & & 0.943 \\
\hline Large artery atherosclerosis & $49(8.2)$ & $547(91.8)$ & 0.440 \\
\hline Cardioembolic stroke & $5(5.6)$ & $85(94.4)$ & 0.203 \\
\hline Small vessel disease & $4(1.8)$ & $221(98.2)$ & 0.740 \\
\hline Other clear causes & $2(2.4)$ & $82(97.6)$ & 0.845 \\
\hline Multiple potential causes & $18(11.8)$ & $135(88.2)$ & 0.999 \\
\hline Unclear cause & $2(6.9)$ & $27(93.1)$ & 0.426 \\
\hline Incomplete work up & $10(5.7)$ & $165(94.3)$ & 0.823 \\
\hline Missing & $3(7.3)$ & $38(92.7)$ & \\
\hline Adherence to medication & & & 0.833 \\
\hline Yes & $81(7.2)$ & $1,046(92.8)$ & \\
\hline No & $13(4.9)$ & $255(95.1)$ & \\
\hline \multicolumn{4}{|l|}{ Values are mean \pm SD or $n(\%)$} \\
\hline
\end{tabular}

$0.139-0.743 ; p=0.008)$, atrial fibrillation (OR 3.866; $95 \%$ CI 1.060-14.104; $p=0.041$ ), smoking (OR 1.544; 95\% CI $1.002-2.379 ; p=0.049)$, TOAST type of patients with unclear cause (OR 2.375; 95\% CI 1.147-4.918; $p=0.020$ ), and NIHSS score at admission (OR 1.092; 95\% CI 1.032$1.155 ; p=0.002)$. Fisher's exact test found that recurrence had no association with adherence to medication ( $p=$ 0.833). Multivariate analysis found that NIHSS score at admission was significantly associated with recurrence (OR 1.088; 95\% CI 1.028-1 152; $p=0.004$ ).

\section{Discussion}

Results from the current study in northern Chinese population showed that men appeared to be more common in young adult ischemic stroke patients. The most common causes of young stroke were large vessel atherosclerosis and small vessel occlusion, followed by cardioembolism. The severity of stroke at admission in young adults was usually mild. The NIHSS score at admission were associated with recurrence of young stroke.
In a previous study, the most common risk factors identified in young adults with ischemic stroke were abnormal lipid metabolism (52.7\%), smoking (47.3\%), hypertension $(39.3 \%)$, and a patent foramen ovale $(32.8 \%)$ [2]. The causes of stroke in this study were estimated as large artery atherosclerosis in $11.3 \%$, small vessel occlusion in $8 \%$, cardioembolism in $24 \%$, other determined etiology in $27.3 \%$, and undetermined etiology in $29.3 \%$. Other available studies reported that cardioembolism accounted 9.25-25\% etiology of young adults ischemic stroke $[9,10]$. In contrast, the present study found that cardioembolism associated with a patent foramen ovale was only observed in a small proportion of patients, with large artery atherosclerosis in a relatively high proportion of patients. The proportions of ischemic stroke due to other causes were comparable with previously reported studies. Therefore, the main causes of stroke in young adults in northern Chinese population seem to be vascular disease, especially large vessel atherosclerosis. We surmise that this finding is due to the large proportion of subjects who had undergone vascular investigations $(1,328 / 1,395)$, and because atherosclerosis has a higher 
prevalence in northern China than in other regions of China.

Cardioembolism is associated with prosthetic heart valves, mitral stenosis, infectious and non-infectious endocarditis, dilated cardiomyopathy, intraluminal thrombosis, and cardiac tumors such as myxoma [11]. Other conditions such as a patent foramen ovale, atrial septal aneurysm, abnormal wall motion, and mitral valve prolapse may also be associated with cardioembolism. It has been suggested that patients with stroke caused by cardioembolism should undergo dynamic electrocardiography to exclude paroxysmal atrial fibrillation, but the detection rate is low (5\%) [12]. Prolongation of the monitoring time may increase the detection rate (6\%) [13]. In this retrospective study, echocardiography was performed in 703/1,395 patients, and electrocardiography and dynamic electrocardiography were performed in $940 / 1,395$ patients. The low proportion of patients diagnosed with cardioembolism was probably associated with the lack of these investigations in all patients. It should be noted that although a patent foramen ovale increases the risk of stroke, it does not cause embolism, and the source of embolism should be identified [14]. Some patients with stroke may therefore have been incorrectly diagnosed with cardioembolism [15], resulting in differences in the reported rates of cardioembolism among studies.

Many studies have investigated the factors associated with stroke recurrence. A recent study reported that age and diabetes mellitus were long-term risk factors for stroke recurrence [16]. Another study reported that a family history of stroke, migraine with aura, anti-phospholipid antibody syndrome, antihypertensive medications, and irregular ingestion of antiplatelet medication increased the risk of recurrence in young adults with stroke [17]. Other studies found that patients with stroke caused by cardioembolism had a high risk of recurrence $[5,18]$. In this study, cardioembolic stroke had no association with recurrence. Our data showed that the major embolic cause was presented as patent foramen ovale, which may have led to inconsistent results with other studies in different regions. We also found that NIHSS score at admission was associated with recurrence of young ischemic stroke. The US NIHSS score is frequently used to describe the severity of stroke and can be used to predict outcome [19]. A high NIHSS score is associated with a high risk of complications [19]. Chumbler et al. [20] reported that the NIHSS score at presentation was associated with the risk of pneumonia in patients with stroke.

This retrospective study is limited by incomplete data in the medical records. This makes it difficult to compare our data with previous studies. Furthermore, the findings of this single-center study may not be generalizable, and further multicenter studies should be performed.

In summary, vascular disease especially premature atherosclerosis is the major risk factor for ischemic stroke in the young adult population of northern China. Timely screening of the cause of stroke with severe NIHSS score needs further attention.

\section{Acknowledgments}

This study was funded by The Clinical Ability Construction Programs of Liaoning Province (LNCCC-D25-2015).

\section{Disclosure Statement}

The authors have no conflicts of interest to disclose.

\section{References}

1 Williams LS, Garg BP, Cohen M, Fleck JD, Biller J: Subtypes of ischemic stroke in children and young adults. Neurology 1997;49: 1541-1545.

2 Renna R, Pilato F, Profice P, Della Marca G, Broccolini A, Morosetti R, Frisullo G, Rossi E, De Stefano V, Di Lazzaro V: Risk factor and etiology analysis of ischemic stroke in young adult patients. J Stroke Cerebrovasc Dis 2014; 23:e221-e227.

3 Naess H, Nyland HI, Thomassen L, Aarseth J, Nyland G, Myhr KM:Incidence and short-term outcome of cerebral infarction in young adults in western Norway. Stroke 2002;33:2105-2108.
4 Greisenegger S, Zehetmayer S, Ferrari J, Lang W, Fizek J, Auff E, Lalouschek W, Serles W: Clinical predictors of death in young and middle-aged patients with ischemic stroke or transient ischemic attack: long-term results of the Vienna Stroke Registry: clinical predictors of ischemic stroke mortality in patients $<60$ years. J Neurol 2011;258:1105-1113.

5 Rutten-Jacobs LC, Maaijwee NA, Arntz RM, Schoonderwaldt HC, Dorresteijn LD, van der Vlugt MJ, van Dijk EJ, de Leeuw FE: Longterm risk of recurrent vascular events after young stroke: the FUTURE study. Ann Neurol 2013;74:592-601.
6 Fromm A, Haaland OA, Naess H, Thomassen L, Waje-Andreassen U: Risk factors and their impact on carotid intima-media thickness in young and middle-aged ischemic stroke patients and controls: the Norwegian Stroke in the Young Study. BMC Res Notes 2014;7:176

7 Jiang B, Ryan KA, Hamedani A, Cheng Y, Sparks MJ, Koontz D, Bean CJ, Gallagher M, Hooper WC, McArdle PF, et al: Prothrombin G20210A mutation is associated with youngonset stroke: the genetics of early-onset stroke study and meta-analysis. Stroke 2014;45:961967.
Characteristics and Risk Factors for

Recurrence in Young Ischemic Stroke
Eur Neurol 2017;77:115-122 DOI: $10.1159 / 000455093$ 
8 Singhal AB, Biller J, Elkind MS, Fullerton HJ, Jauch EC, Kittner SJ, Levine DA, Levine SR: Recognition and management of stroke in young adults and adolescents. Neurology 2013;81:1089-1097.

9 Jovicevic M, Divjak I, Slankamenac P, Bozic K, Zikic TR, Kaloci SR: [The most frequent causes of ischemic stroke in young adults]. Med Pregl 2011;64:331-335.

10 Jaffre A, Ruidavets JB, Calviere L, Viguier A, Ferrieres J, Larrue V: Risk factor profile by etiological subtype of ischemic stroke in the young. Clin Neurol Neurosurg 2014;120:7883.

11 Gegouskov V, Kadner A, Engelberger L, Carrel T, Tevaearai $\mathrm{H}$ : Papillary fibroelastoma of the heart. Heart Surg Forum 2008;11:E333E339.

12 Liao J, Khalid Z, Scallan C, Morillo C, O'Donnell M: Noninvasive cardiac monitoring for detecting paroxysmal atrial fibrillation or flutter after acute ischemic stroke: a systematic review. Stroke 2007;38:2935-2940.

13 Jabaudon D, Sztajzel J, Sievert K, Landis T, Sztajzel R: Usefulness of ambulatory 7-day ECG monitoring for the detection of atrial fibrillation and flutter after acute stroke and transient ischemic attack. Stroke 2004;35: 1647-1651.

14 Erharhaghen J, Bartz M, Di Giovanni S, Melms A, Haarmeier T, Sieverding L: An unusual location of deep venous thrombosis associated with ischemic stroke and persistent foramen ovale. Case Rep Neurol 2011;3:160164.

15 Ferro JM, Massaro AR, Mas JL: Aetiological diagnosis of ischaemic stroke in young adults. Lancet Neurol 2010;9:1085-1096.

16 Pennlert J, Eriksson M, Carlberg B, Wiklund PG: Long-term risk and predictors of recurrent stroke beyond the acute phase. Stroke 2014;45:1839-1841.
17 Boone M, Chillon JM, Garcia PY, Canaple S, Lamy C, Godefroy O, Bugnicourt JM: NIHSS and acute complications after anterior and posterior circulation strokes. Ther Clin Risk Manag 2012;8:87-93.

18 Toni D, Di Angelantonio E, Di Mascio MT, Vinisko R, Bath PM; PRoFESS Study Group: Types of stroke recurrence in patients with ischemic stroke: a substudy from the PRoFESS trial. Int J Stroke 2014;9:873-878.

19 Hand B, Page SJ, White S: Stroke survivors scoring zero on the NIH stroke scale score still exhibit significant motor impairment and functional limitation. Stroke Res Treat 2014; 2014:462681.

20 Chumbler NR, Williams LS, Wells CK, Lo AC, Nadeau S, Peixoto AJ, Gorman M, Boice JL, Concato J, Bravata DM: Derivation and validation of a clinical system for predicting pneumonia in acute stroke. Neuroepidemiology 2010;34:193-199. 